\title{
The enigmatic function of chandelier cells
}

\author{
Alan R. Woodruff ${ }^{*}$, Stewart A. Anderson ${ }^{2}$ and Rafael Yuste ${ }^{1}$ \\ 1 Department of Biological Sciences, Howard Hughes Medical Institute, Columbia University, New York, NY, USA \\ 2 Department of Psychiatry, Weill Cornell Medical College, Cornell University, New York, NY, USA
}

Chandelier (or axo-axonic) cells are one of the most distinctive GABAergic interneurons in the brain. Their exquisite target specificity for the axon initial segment of pyramidal neurons, together with their GABAergic nature, long suggested the possibility that they provide the ultimate inhibitory control of pyramidal neuron output. Recent findings indicate that their function may be more complicated, and perhaps more interesting, than initially believed. Here we review these recent developments and their implications. We focus in particular on whether chandelier cells may provide a depolarizing, excitatory effect on pyramidal neuron output, in addition to a powerful inhibition.

Keywords: GABAergic depolarization, excitation, cortex, axon initial segment

\section{INTRODUCTION}

The mammalian brain is an organ of seemingly impossible complexity. There are billions of neurons, trillions of synaptic connections, and probably hundreds of distinct cell types, each of which is presumably specialized to perform distinct tasks. A crucial step in addressing the underlying logic in the design of the brain is therefore identifying the function, or functions, of a given neuronal class.

The most obvious separation in neuron function within cortical structures is between excitatory, glutamatergic pyramidal (or principal) neurons and inhibitory GABAergic interneurons. Information is presumably carried and processed by pyramidal (Pyr) neurons, whose connections traverse cortical layers and regions. Interneurons, by contrast, are typically considered to project only locally, and provide a means of controlling the excitation provided by pyramidal neurons. It is, of course, not that simple. It turns out that a vast heterogeneity exists amongst the GABAergic interneurons, which constitute $\sim 20 \%$ of all cortical neurons. This heterogeneity has long been recognized (Ramón y Cajal, 1899; Lorente de Nó, 1922), and one of the first aspects of this heterogeneity to be noticed, their "short axons," is likely to be one of the most important-the distinct axonal arborizations of different interneurons reflects their subcellular target specificity, which in turn has important implications for their function. Indeed, a common delineation exists between interneurons targeting dendritic domains, such as neocortical Martinotti and double bouquet cells, and those targeting axo-somatic domains, such as chandelier and basket cells (Markram et al., 2004). Whereas dendrite-targeting neurons may be more suited to modify and gate incoming excitatory input (Murayama et al., 2009), axo-somatic interneurons are likely to exhibit a greater impact on the direct output of the postsynaptic neuron (Marr, 1970; Miles et al., 1996).

Here we focus on attempting to understand the chandelier cell (ChCs), an interneuron type that exemplifies the target specificity of cortical interneurons and thus potentially illustrates the purposeful design of neuronal circuits. ChCs were "missed" by Cajal and Lorente and were first identified by Szentagothai and, independently, by Jones (Jones, 1975; Szentagothai, 1975). The defining morphological characteristic of ChCs is the array of short, vertically oriented rows of terminal boutons, which resemble candlesticks. Originally believed to contact the apical dendrite of pyramidal cells (Szentagothai, 1975), these axonal cartridges were later shown via electron microscopic 


\section{Chandelier cell}

GABAergic interneuron whose exclusive postsynaptic target is the axon initial segment of pyramidal neurons. reconstructions of Golgi-stained specimens to exclusively contact the axon initial segment (AIS) of pyramidal neurons (Figure 1C; Somogyi, 1977; Fairen and Valverde, 1980). Because of this, chandelier neurons were renamed "axo-axonic cells," a nomenclature that is used interchangeably. This highly stereotyped and visually striking appearance of ChCs facilitated early studies regarding their location, abundance, and neurochemical features. ChCs are present in all cortical layers,
A

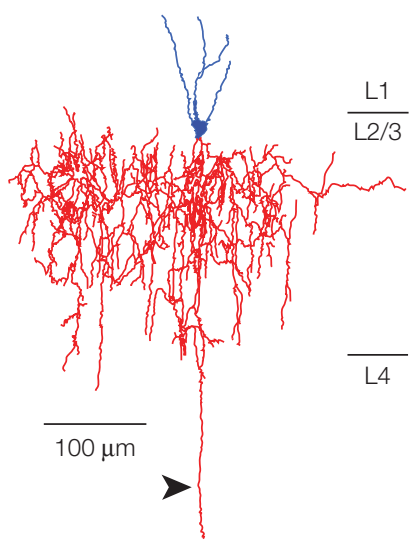

C

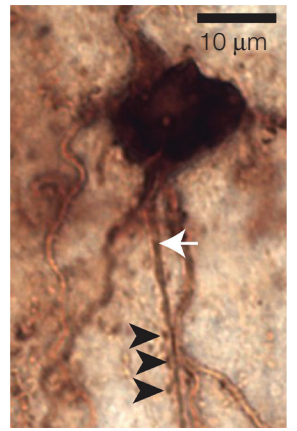

E

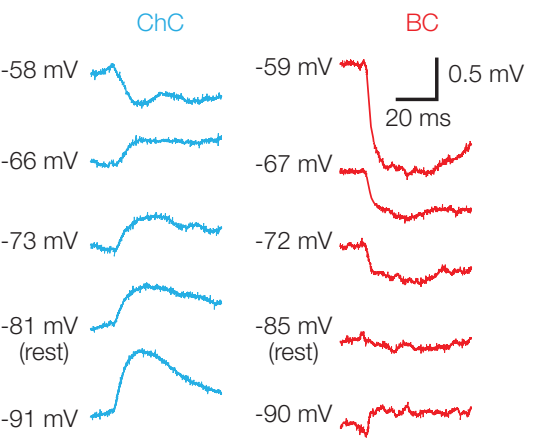

B

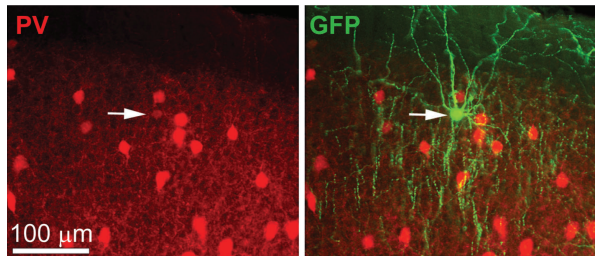

D
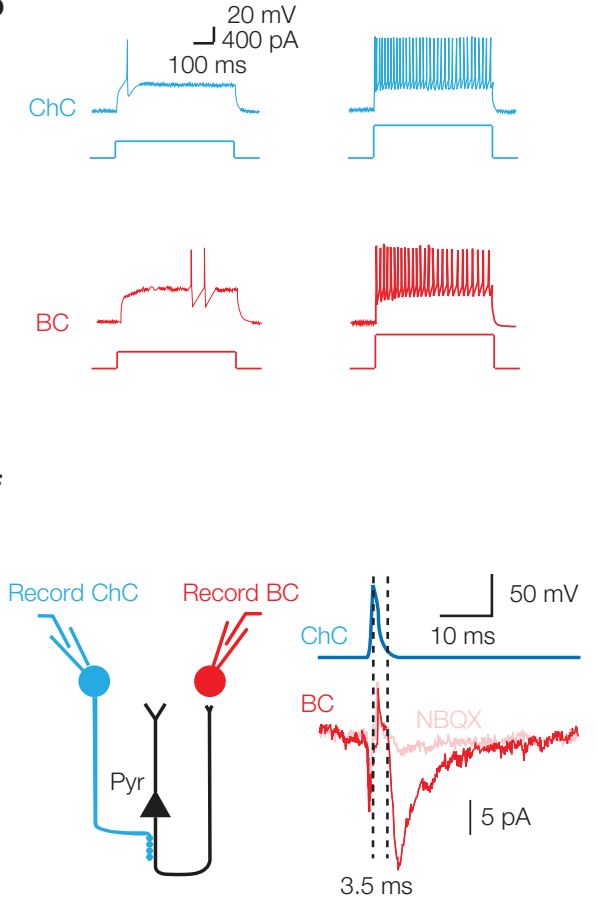

FIGURE 1 | Characteristic features of chandelier cells. (A) Neurolucida reconstruction of a layer 2/3 chandelier cell. Cell body and dendrites are in blue, axon in red. Several vertically oriented axonal segments are visible, and are the characteristic morphological feature of ChCs. The long descending axon (arrowhead) reached and arborized in layer 6, but has been digitally truncated. (B) Parvalbumin immunoreactivity of a ChC. A GFP-labeled ChC (right panel, arrow) co-expresses PV (left panel, arrow). (C) Biocytin-filled ChC forms a row of cartridge synapses (arrowheads) on the AIS of a biocytin-filled cortical pyramidal neuron (axon marked by white arrow). (D) Chandelier and basket cells have characteristic responses to threshold current injection. Both cells exhibit the fast-spiking phenotype at higher current intensities (right panel, $2 \times$ threshold illustrated). (E) In cortical layer $2 / 3$ pyramids, $E_{G A B A}$ differs at ChC-Pyr and BC-Pyr synapses. (F) ChCs can initiate polysynaptic events. Activation of a GABAergic $\mathrm{ChC}$ evokes a response in a simultaneously recorded basket cell. The response (left panel) is sensitive to the AMPA receptor antagonist NBOX, has a disynaptic latency, and is observed in a cell type $(\mathrm{BC})$ that receives no direct synapses from ChCs. Schematic of disynaptic circuit is shown in right panel. (B, D and E) modified, from Woodruff et al. (2009). 
most abundantly in layer 2/3 (DeFelipe et al., 1985; Inda et al., 2007). Although originally described in neocortex (Szentagothai, 1975), ChCs have also been found in the CA3 (Sik et al., 1993), CA1 (Somogyi et al., 1983) and dentate gyrus (Soriano and Frotscher, 1989) regions of the hippocampus, and in the amygdala (McDonald, 1982). The expression of GABA (Somogyi et al., 1985), parvalbumin (PV; DeFelipe et al., 1989), and corticotropin-releasing factor (Lewis et al., 1989) provides a neurochemical identity to these neurons (Figure 1B).

Despite this knowledge, it has been significantly more difficult to ascertain the physiological properties of ChCs, and more particularly their putative functions. This is in part due to the rarity of ChCs and the fact that common markers label both chandelier and the much more abundant basket cells. To date there is currently no known unique ChC marker. Although a variety of transgenic mouse lines now exist in which various populations of interneurons are fluorescently labeled, including PV-positive neurons (Meyer et al., 2002; Chattopadhyaya et al., 2004), ChCs have been recorded from much less frequently than have the more numerous basket cells. Physiologically, both ChCs and BCs fire high frequency, minimally adapting trains of narrow action potentials - a fast-spiking phenotype. In the cortex, BCs and ChCs were believed indistinguishable on physiological grounds (Kawaguchi, 1995; Gonzalez-Burgos et al., 2005), while in the hippocampus, ChCs were reported to exhibit a greater degree of spike frequency adaptation than BCs (Han, 1994). Thus in most cases, unequivocal identification of a $\mathrm{ChC}$ was only possible following post-recording recovery of the neuron's morphology (Figure 1A). Whilst this is not a difficult procedure, it is not routinely performed, and the number of physiological and functional studies on ChCs has been somewhat limited.

The studies hinting at a functional role for ChCs are therefore correlative rather than causative. In vivo recordings have revealed the firing pattern of hippocampal ChCs during various network states (Klausberger et al., 2003; Tukker et al., 2007), showing that ChCs fire antiphase to pyramidal neurons during theta activity, and fire immediately prior to pyramidal neuron activation during sharp-wave-associated ripples (Klausberger et al., 2003). Perhaps a more direct functional role was suggested by whole-cell recordings from $\mathrm{ChCs}$ in rat somatosensory cortex during whisker deflection. Whisker-evoked stimulation revealed different sequences of afferent input compared to BCs (Zhu et al., 2004), and resulted in ChCs being less receptive to ascending sensory input. However, ChCs markedly increased their output during conditions of high network activity, implying that ChCs may be recruited to dampen excessive excitation. Until recently, therefore, all evidence seemed to suggest that ChCs were inhibitory GABAergic neurons, unique in their high degree of spatial target selectivity, but otherwise not particularly remarkable in comparison to other GABAergic cell types.

\section{ARE CHANDELIER CELLS DEPOLARIZING?}

In 2006, a controversial paper appeared demonstrating an excitatory effect of cortical layer $2 / 3$ chandelier cell activation (Szabadics et al., 2006). Three pieces of evidence were presented in support of this. Firstly, disynaptic, glutamatergic excitatory postsynaptic potentials (EPSPs) could be elicited following a single spike in the chandelier cell - an effect attributed to direct recruitment of pyramidal neurons by ChCs (Figure 1F). But for direct recruitment of a pyramidal neuron to occur with such a short latency, the $\mathrm{ChC}$ synapse at the AIS had to be depolarizing, a condition not previously considered for this particular synapse. Secondly, the authors confirmed that the ChC synapse was indeed depolarizing, with the GABA reversal potential $\left(E_{\mathrm{GABA}}\right)$ significantly elevated above the neuron's resting potential $\left(V_{\text {rest }}\right)$, and above that of basket cell synapses (Figure 1E). Importantly, $E_{\mathrm{GABA}}$ in this study was measured using the antibiotic gramicidin in the postsynaptic pipette of a paired recording. Gramicidin forms membrane pores that allow exclusive exchange of monovalent cations and small uncharged molecules (Kyrozis and Reichling, 1995). Thus there is no dialysis of intracellular chloride, as occurs with whole-cell patching, and more physiological $E_{\mathrm{GABA}}$ responses are recorded through the perforated neuronal membrane. Thirdly, immunogold labeling and electron microscopic reconstruction demonstrated an apparent absence of the potassium-chloride cotransporter KCC2 from the AIS - an effect that would theoretically lead to less extrusion of intracellular chloride and lead to the depolarized $E_{\mathrm{GABA}}$ reported.

Thus, a powerful form of excitation had been demonstrated for a neuron previously believed based on the strategic location of its GABAergic synapses - to exert a powerful form of inhibition. The Szabadics data did not exclude an inhibitory action of ChCs, but rather added an excitatory role to their function. Instead of being purely inhibitory, ChCs now had the potential to be either inhibitory or excitatory, depending on the membrane potential of the postsynaptic neuron. 
By extension, the overall activity state of the network would determine whether ChCs behaved as excitatory or inhibitory neurons.

Further support for a depolarizing effect fol-

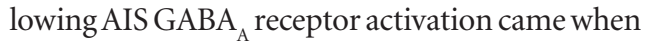
it was shown, again using gramicidin-based patch recordings, that dentate granule cells exhibited an axo-somato-dendritic gradient in $E_{\mathrm{GABA}}$ (Khirug et al., 2008). The relatively depolarized axonal $E_{\text {GABA }}$ was shown to persist, to some degree, even in whole-cell recordings, an effect attributed to efficient import of chloride by the $\mathrm{Na}-\mathrm{K}-2 \mathrm{Cl}$ cotransporter NKCC1 (Khirug et al., 2008), rather than, or perhaps in addition to, the absence of KCC2 suggested by Szabadics et al. (2006). A similar hyperpolarizing-depolarizing $E_{\mathrm{GABA}}$ gradient from dendrite to axon was shown for cortical pyramidal cells of layer $2 / 3$.
Both the Szabadics and Khirug data were obtained primarily using the gramicidin perforated patch technique. Although avoiding direct chloride exchange between pipette and cell, gramicidin recordings may still be prone to some error in calculating $E_{\mathrm{GABA}}$. This is because the chloride equilibrium potential is set by the combined activity of the cation chloride cotransporters KCC2 and NKCC1 (Figure 2A). Because both sodium and potassium permeate the membrane pores created by gramicidin, pipette concentrations of these ions that don't precisely match the physiological intracellular concentrations can alter the activity of the transporters, and consequently shift $E_{\mathrm{GABA}}$, potentially giving a spurious reading. For example, resting $\left[\mathrm{Na}^{+}\right]_{\mathrm{i}}$ is low relative to resting $\left[\mathrm{K}^{+}\right]_{i}$, so slight inaccuracies in pipette $\left[\mathrm{Na}^{+}\right]$in particular may produce significant effects

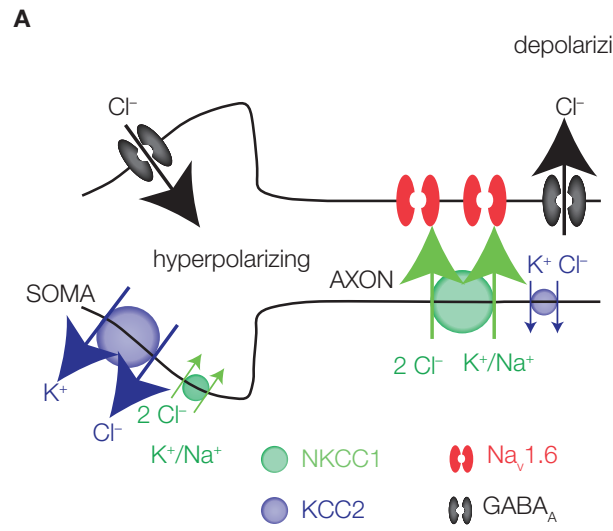

C
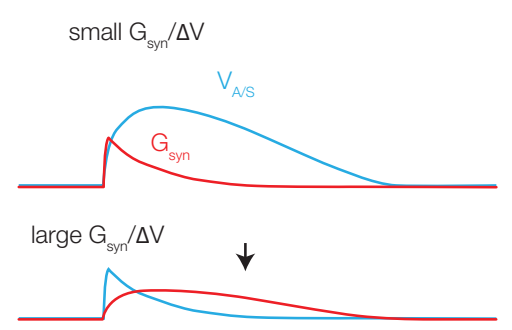
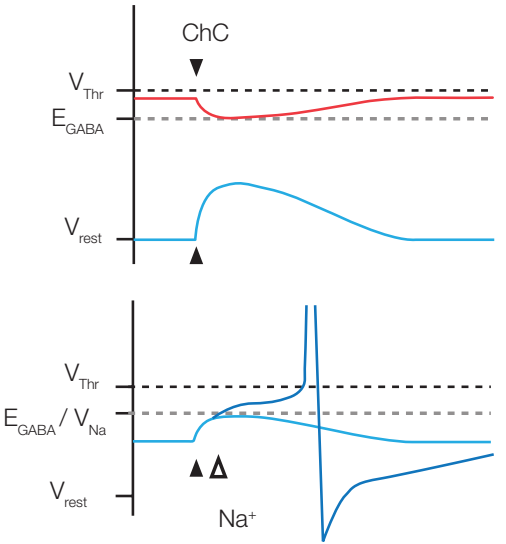

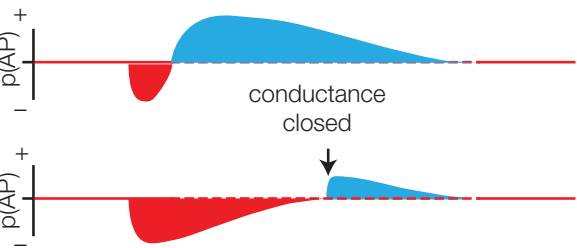

FIGURE 2 | GABAergic excitation. (A) The soma and axon of a pyramidal neuron are represented. Somatic $E_{\mathrm{Cl}}$ is hyperpolarized due to the high expression of KCC2 and low expression of NKCC1. GABA receptor activation leads to hyperpolarization, or no net flux of ions (shunting). At the AIS, the expression of cation chloride transporters is reversed, favoring chloride efflux and depolarization upon GABA channel opening. Low-threshold NaV1.6 sodium channels may be activated by the depolarization, enhancing the excitatory effect. (B) Although depolarizing, a GABAergic event may be inhibitory, due to the conductance effect of channel opening. However, the inhibitory conductance effect (red) decays more quickly than the excitatory membrane potential change (blue), providing at least some window of excitation (right panel, bottom). The strength and duration of excitation (blue) and inhibition (red), denoted here as spike probability ( $p(A P)$ ), will depend on the magnitude of the conductance effect relative to the change in membrane potential. (C) The ChC synapse is expected to be hyperpolarizing during high activity periods (red) and depolarizing under resting conditions. Because AIS $E_{\text {GABA }}$ is in the range of $\mathrm{Na}^{+}$channel activation, some depolarizations may be enhanced by sodium currents (bottom panel). 
on transporter activity. For this reason, regions of the neuron where sodium exchange via NKCC1 is used to set the transmembrane chloride gradient, such as the axon (Khirug et al., 2008), may be more prone to allowing inaccurate values of $E_{\mathrm{GABA}}$ to be recorded. Additionally, slight changes in $\left[\mathrm{Na}^{+}\right]_{\mathrm{i}}$ and $\left[\mathrm{K}^{+}\right]_{\mathrm{i}}$ introduced by the recording pipette may alter the neuron's resting membrane potential, causing a compensatory redistribution of chloride ions across the membrane through passive diffusion.

These concerns were addressed using a novel, non-invasive approach to recording the polarity of unitary GABAergic responses in hippocampal CA1 (Glickfeld et al., 2009). The authors recorded "unitary fields" - i.e., extracellular field potentials produced by activation of a single, morphologically verified interneuron - at various somatodendritic locations of the CA1 pyramidal cell population. These recordings rely on small changes in the flow of ions around the extracellular recording electrode. As membrane channels open, transmembrane ion flux results in accumulations and depletions of the permeant ions in the regions proximal to the recording electrode. The direction of current flow is indicated by the polarity of the extracellular response. Importantly, this technique does not interfere in any way with the intracellular milieu of the postsynaptic neurons, and in this respect represents an improvement on whole-cell and gramicidin recordings. For each class of interneuron recorded, targeting either the dendritic, somatic, or axonal compartment, the authors found only a hyperpolarizing effect. Thus the non-invasive approach taken provided no evidence for a depolarizing effect of ChCs.

A potential caveat with the technique arises, however, because Glickfeld et al. (2009) used very high (molar) concentrations of chloride ions in their extracellular recording electrodes, which may have leaked into the surrounding neuropil. Typically, extracellular chloride concentrations

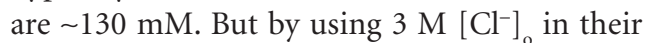
extracellular pipette, the authors could potentially have produced a $\sim 20$-fold increase in local chloride concentrations, should any chloride leakage occur from the pipette solution. Increased extracellular chloride levels would shift $E_{\mathrm{GABA}}$ to hyperpolarized potentials, favoring chloride influx upon GABA $_{A}$ channel opening, and thus a hyperpolarizing response at the synapse. Could the hyperpolarizing response of CA1 ChCs, which contrasts with the gramicidin recordings in cortex and in hippocampal dentate granule cells, therefore be an artifact of the recording technique? If significant chloride leak did occur, any changes in $\left[\mathrm{Cl}^{-}\right]_{\mathrm{o}}$ at the site of the synapse may be expected to dissipate if the recording pipette is moved hundreds of microns away, along the somatodendritic axis. Consistent with this, when the recording pipette was moved to distal dendritic sites, the authors still observed unitary fields of a polarity consistent with a hyperpolarizing synapse at the AIS. Thus even when not subjected to the possibility of chloride leak from the recording pipette, the synapse was hyperpolarizing. Additionally, the concern of $\mathrm{Cl}^{-}$leaking from the electrode was addressed by replacing the $3 \mathrm{M} \mathrm{NaCl}$ in the recording pipette with artificial cerebrospinal fluid (ACSF). This manipulation generated similar results in the observed amplitude of the unitary field evoked by basket synapses (Glickfeld et al., 2009), which would not be expected if chloride leak was responsible for a hyperpolarizing shift in $E_{\mathrm{GABA}}$.

These data, therefore, obtained using a noninvasive technique (unitary field recordings), are at odds with the data of Szabadics et al. (2006) and Khirug et al. (2008), both of which were obtained using the slightly more invasive gramicidin perforated patch technique. The polarity of effect of a GABAergic synapse is likely a major indicator and determinant of that synapse's functional role. The conflicting data described above, and the differing techniques used to obtain the different results, prompted us to investigate whether the depolarizing $\mathrm{ChC}$ effect may be an artifact introduced by gramicidin recordings.

\section{DEPOLARIZING CHANDELIERS, REVISITED}

The goal of our experiments (Woodruff et al., 2009) was to re-examine the depolarizing effect of cortical ChCs. For this, we first found a method to routinely record from $\mathrm{ChCs}$, something that to our knowledge had not previously been achieved. We used Nkx2.1Cre MADM mice, a strain of genetically engineered animals that express GFP in a subset of neocortical interneurons arising from the medial ganglionic eminence. This subset included ChCs, which importantly were labeled brightly, allowing the distinctive axonal cartridges of ChCs to be identified prior to recordings. ChCs could therefore be easily targeted, particularly in upper layer $2 / 3$, near the layer 1 border. We routinely recorded from $\mathrm{ChCs}$ in layer $2 / 3$ and were able to distinguish them from basket cells based on their firing pattern and passive membrane properties (Figure 1D; Woodruff et al., 2009). This distinction was extremely reliable, as confirmed by the subsequent anatomical identification of recorded cells, and obviated the need for morphological verification in the vast majority of subsequent cases.
The electrical potential produced by neurons due to transmembrane ion flux, measured extracellularly. 
We first performed paired recordings from presynaptic ChCs and gramicidin-patched postsynaptic pyramidal cells. Our data, taken from mouse neocortex, closely matched that of Szabadics et al. (2006) from rat neocortex, with $E_{\mathrm{GABA}}$ of the ChC synapse lying $\sim 20 \mathrm{mV}$ above resting membrane potential, and $\sim 10 \mathrm{mV}$ below action potential threshold. Similar experiments performed on basket cell synapses showed a predominantly shunting synapse, in which $E_{\mathrm{GABA}}$ was typically at the neuron's resting membrane potential. While this was consistent with the previous gramicidin-based recordings from Szabadics and Khirug, the possibility remained that the depolarized axonal $E_{\mathrm{GABA}}$ was simply the result of changes in the activity of NKCC1 that may occur via alterations in intracellular $\mathrm{Na}^{+}$concentrations, as outlined above. We therefore complemented these recordings with a completely non-invasive technique, tight seal cell-attached recordings. Cell-attached recordings have only rarely been used to record synaptic potentials (but see Kantrowitz et al., 2005; Perkins, 2006), and to our knowledge, only to record largeamplitude, network driven events (Kantrowitz et al., 2005). We first showed that detecting submillivolt voltage deflections would be possible with tight seal cell-attached recordings. Our paired recordings then consistently revealed a depolarization of the postsynaptic pyramidal cell after $\mathrm{ChC}$ activation. All recordings, regardless of whether they displayed evidence of a connection in the cell-attached configuration, were subsequently tested in whole-cell mode for the presence of a synaptic response. We were thus able to distinguish purely shunting synapses, which would not be detectable in cell-attached mode but would be revealed upon entering wholecell mode, from unconnected ChC-Pyr pairs. Importantly, we never observed purely shunting or hyperpolarizing ChC-Pyr synapses. These data supported our gramicidin recordings, and furthermore demonstrated that the ChC-evoked depolarization at the AIS observed using gramicidin recordings could not be disregarded as an experimental artifact.

These data demonstrated that at least for layer 2/3 cortical ChCs, and seemingly also for dentate granule cells, GABA at the AIS is depolarizing. However, ChCs in CA1 produce a hyperpolarization. Because the reversal potential of a GABAergic synapse is likely an important determinant of that synapse's function, an interesting dichotomy may exist in the roles played by ChCs in different parts of the brain, an intriguing possibility for a cell type previously hypothesized to be a fine example of functional specialization.

\section{CAN CORTICAL CHANDELIER CELLS BE EXCITATORY?}

But does the axonal depolarization caused by cortical ChCs promote or inhibit action potential firing? To date, the only demonstration that $\mathrm{ChCs}$ are excitatory - that they promote firing - is the suprathreshold activation of pyramidal neurons reported by several groups (Szabadics et al., 2006; Woodruff et al., 2006; Molnar et al., 2008; Glickfeld et al., 2009). But even this phenomenon, while clearly excitatory, should be interpreted cautiously.

Firstly, nerve injury is known to result in an upregulation of NKCC1 and a downregulation of KCC2 (Hasbargen et al., 2010), both of which would favor a depolarized $E_{\mathrm{GABA}}$. Thus the frequent axotomies that occur during the preparation of brain slices could create artificially high axonal $E_{\mathrm{GABA}}$ values, leading to hyperexcitability.

Secondly, chandelier-triggered activation of a pyramidal neuron has never been directly observed, instead being inferred by the presence of a disynaptic, glutamatergic response that is time-locked to the $\mathrm{ChC}$ spike (Figure 1F) and which is sensitive to the GABA receptor antagonist bicuculline. The reason for this may be merely statistical, in that the chances of recording from the one or two responsive pyramidal neurons are low. Alternatively, it may be because the active pyramidal neurons are unhealthy, with a consequent increase in $\mathrm{NKCC} 1$ expression responsible for the phenomenon. These neurons would not be targeted for patching. Bulk loading of membranepermeable acetoxymethyl (AM) $\mathrm{Ca}^{2+}$ indicators, which allows action potentials to be monitored in hundreds of neurons (Yuste and Katz, 1991; Smetters et al., 1999), should facilitate the detection of active neurons, although we remain unaware of success with this technique. This failure could be due to low signal-to-noise, to unhealthy neurons not incorporating the $\mathrm{AM} \mathrm{Ca}{ }^{2+}$ indicators, or to saturating concentrations of indicator that prevent the detection of action potentialinduced fluorescence changes. Alternatively, the action potential produced by chandelier activation may, depending on its initiation point relative to the ChC synapse, fail to backpropagate to the soma. This would prevent its detection both by somatic patch recording and somatic actionpotential derived fluorescence changes.

It is certainly not clear that the suprathreshold activation described by us and others - which is the sole excitatory $\mathrm{ChC}$ action reported to date - is a pathological response. However, until such activations are directly observed by recording from a healthy pyramidal neuron, or until it is observed in the intact animal, the possibility that they are artifactual should be kept in mind. 
Disregarding the suprathreshold activations induced by ChCs, we are left with the fact that in some parts of the brain, but perhaps not in others, ChCs are depolarizing. This subthreshold depolarization is, in fact, likely to be the effect felt by the vast majority of recipient pyramidal neurons upon ChC activation. A highly pertinent question, then, is whether this subthreshold depolarization is excitatory. A few factors merit some thought. The opening of any membrane channel decreases the neuronal input resistance, causing shunting inhibition - a given current then produces a smaller voltage deflection. This shunting effect is therefore by nature inhibitory, and antagonistic to any depolarization (Figure 2C). Whether the magnitude of the conductance shunt outweighs the magnitude of the depolarization is therefore an important consideration, though it should be noted that once the conductance closes, any remaining depolarization is likely to be excitatory (Figure 2C).

Secondly, $E_{\mathrm{GABA}}$ for the ChC synapse lies below threshold. Although a ChC PSP may initially be depolarizing, with the pyramidal neuron below $E_{\mathrm{GABA}}$, continued depolarization of the pyramid above $E_{\mathrm{GABA}}$, perhaps through additional glutamatergic inputs, will result in the ChC PSP switching to hyperpolarizing and inhibitory, provided the conductance is still open. Whether the net effect of ChC activation under these conditions will help or hinder firing is therefore not straightforward.

Another point worth considering is that conductance effects aside, there still exists the theoretical possibility for a depolarization-induced inhibition due to inactivation of the $\mathrm{Na}^{+}$channels responsible for spike generation. However, we believe this is unlikely to be a significant factor in the case of ChCs, given that depolarization also greatly enhances $\mathrm{Na}^{+}$channel open probability. Indeed, if this possibility is to be considered, one must also consider the possibility that similar mechanisms can result in glutamatergic inputs being inhibitory due to the depolarization they produce - the situation is analogous. In addition, the AIS contains not only fast-inactivating $\mathrm{Na}^{+}$ channels responsible for spike generation, but also slowly inactivating channels with a negatively shifted activation threshold (Astman et al., 2006), which underlie the "persistent" sodium current,

Shunting inhibition

A form of inhibition that results from the opening of a membrane conductance and the decrease in excitability this causes. Typically used to describe the effect of a GABAergic interneuron when $E_{\mathrm{GABA}}$ of the synapse is equal to the membrane potential of the neuron.
$I_{\mathrm{NAP}}$ (Fleidervish et al., 2010). This persistent current may be important in aiding spike generation following a ChC input.

A situation analogous to that in cortical ChCs, in which $E_{\mathrm{GABA}}$ lies significantly above $V_{\text {rest }}$ but below $V_{\text {Thr }}$, exists in immature neocortical (Rheims et al., 2008) and hippocampal pyramidal cells. Provided the driving force $\left(E_{\mathrm{GABA}}-V_{\text {rest }}\right)$ is high enough, and $E_{\mathrm{GABA}}$ is not too distant from $V_{\mathrm{Thr}}$, GABA is proven to be excitatory in these neurons. The values reported for the three determining parameters in this study are, in fact, in line with those at the cortical ChC synapse, and an important requirement for GABAergic excitation appears to be the activation of voltage-gated sodium channels (VGSCs), which allow the neuron to continue depolarizing despite being above $E_{\mathrm{GABA}}$ (Figures 2A,B; Rheims et al., 2008; Valeeva et al., 2010). Since VGSCs at the AIS have a relatively hyperpolarized activation threshold (Colbert and Pan, 2002; Astman et al., 2006; Hu et al., 2009) due to the presence of $\mathrm{Na}_{\mathrm{v}} 1.6$ channels, in the range of axonal $E_{\mathrm{GABA}}$, it certainly seems possible that ChCs can be excitatory in a manner similar to that seen in immature neurons.

It should be noted that there is considerable neuron to neuron variability in both $E_{\mathrm{GABA}}$ and $V_{\text {Thr }}$. Both of these parameters may be plastic, changing according to the neuron's activity history (Henze and Buzsaki, 2001; Woodin et al., 2003; Fiumelli et al., 2005), and small changes in these values are likely to be critical in determining whether GABA can be excitatory (Rheims et al., 2008). Thus, the precise interplay of the neuron's membrane potential, $E_{\mathrm{GABA}}$ and $V_{\mathrm{Thr}}$, their modification by prior activity, and the activation of axonal VGSCs seem to be the critical parameters that will determine whether ChCs can provide excitatory, as well as inhibitory, input.

The discussion so far has focused on experiments performed in vitro. However, neurons in vivo are constantly bombarded with synaptic input, producing a depolarized and fluctuating membrane potential that promotes the opening and closure of a vast array of voltage dependent channels (Destexhe et al., 2003). That in vivo membrane potentials are considerably more depolarized than in the quiescent in vitro slice may be particularly relevant for ChCs. Axonal $E_{\mathrm{GABA}}$ in cortical layer $2 / 3$ neurons is in the range of -65 to $-55 \mathrm{mV}$, values similar to mean membrane potentials recorded in awake animals (Brecht et al., 2004; Poulet and Petersen, 2008; Gentet et al., 2010). Predicting the effect of ChCs in vivo therefore becomes quite a complicated exercise. If the in vivo membrane potential fluctuates either side of $E_{\mathrm{GABA}}$, the $\mathrm{ChC}$ has the potential to be excitatory as well as inhibitory. This may promote the homogenization of pyramidal neuron firing rates, inhibiting strongly active neurons but exciting those that are less active (Vida et al., 2006). In this scenario, ChCs may act as activity sensors, passively adjusting their function according to what is required by the surrounding 
network. The effect will be dictated, of course, by the pyramidal neuron membrane potential, and by the complex interplay of that neuron's synaptic inputs. Whether the membrane potential ever stays below $E_{\mathrm{GABA}}$ for a sufficient period of time to allow depolarization, and whether the presumably small amount of depolarization (so close to $E_{\mathrm{GABA}}$ ) provided by the ChC will outweigh the shunting effect, are critical questions. Alternatively, perhaps the $\mathrm{ChC}$ synapse in vivo should be considered predominantly shunting, and thus purely inhibitory. Indeed, during active states, the shunting effect often ascribed to basket cells, based on $E_{\mathrm{GABA}}$ approximating resting potential in a slice, may be more appropriate for $\mathrm{ChCs}$, while BCs would instead provide hyperpolarizing inhibition. In this way, ChCs and BCs could have quite distinct inhibitory, perisomatic functions. In CA1, where the ChC and BC synapses presumably have more similar reversal potentials, any differences in function must depend more critically on the location of the synapse (AIS vs. soma/proximal dendrites), or on differences in their pre and postsynaptic partners.

\section{FUTURE DIRECTIONS}

While it has recently become more feasible to record from ChCs, significant barriers to understanding their function remain. Firstly, because of their sparseness, recording from and manipulating more than one or two at a time is still difficult. Although some studies report robust networklevel effects after manipulation of a single neuron (Brecht et al., 2004; Houweling and Brecht, 2008; Bonifazi et al., 2009), it would certainly be beneficial to selectively control several ChCs at once. This sort of control has been demonstrated for PV-expressing fast-spiking cells using neurons transfected with channelrhodopsin-2 (ChR2; Cardin et al., 2009; Sohal et al., 2009), a lightactivated proton pump capable of depolarizing and activating neurons with high temporal resolution (Boyden et al., 2005). As of now, however, no unique genetic marker exists for $\mathrm{ChCs}$, so that interrogating their function in this manner is not yet possible. We expect this to happen in the near future, however, and their currently murky role should become considerably clearer.

Another important direction will be to determine the synaptic partners of ChCs. Their postsynaptic targets are quite well established, predominantly being local pyramidal neurons, although some interlaminar connections also exist (Somogyi et al., 1982). There are increasing numbers of reports demonstrating target specificity in neuronal connections (Yoshimura et al., 2005; Brown and Hestrin, 2009; Anderson et al.,
2010; Varga et al., 2010). Whether there is specificity in which particular local and interlaminar neurons ChCs target is unknown, although there is some evidence that they may preferentially contact pyramidal neurons with predominantly intracortical projections (De Carlos et al., 1985; Farinas and DeFelipe, 1991). The presynaptic partners of ChCs are less well documented. Laser scanning photostimulation (LSPS) was recently applied to L2/3 ChCs in mouse primary somatosensory cortex, demonstrating excitatory input predominantly from layers $2 / 3$ and $5 \mathrm{a}$, and inhibitory input from L2/3 and L1 ( Xu and Callaway, 2009). While this laminar input information is certainly useful, a higher resolution technique such as two-photon photostimulation (Nikolenko et al., 2007) could be useful in determining more precise input specificity. In addition, because these mapping experiments are performed in slices, a great number of longer-range, inter-areal connections are likely to be severed. We commonly find ChCs at the border between layer 1 and layer 2/3, a site from which their dendrites have easy access to long-range "feedback" projections from higher cortical areas. A promising method for determining inter-areal connectivity is monosynaptic, retrograde tracing using a deletion-mutant rabies virus (Wickersham et al., 2007). These projections are unlikely to be maintained in vitro. In theory however, in vivo single-cell electroporation or whole-cell patching of a $\mathrm{ChC}$ at the layer 1 border is possible and should allow the introduction of the virus, enabling both its local and longrange presynaptic connections to be elucidated (Marshel et al., 2010). It would be interesting, for example, to determine the similarity in inter-areal input received by the apical tuft of $\mathrm{L} 2 / 3$ pyramidal neurons and the layer 1 dendrites of $\mathrm{ChCs}$ located in upper layer $2 / 3$, whose major targets are those same pyramidal neurons.

While dissecting the synaptic and network effects of chandelier neurons on cortical activity remains a major challenge, considering how these effects may change during later postnatal development adds another important twist to this story. Like other PV-expressing interneurons, ChCs in the mouse originate in the medial ganglionic eminence from progenitors that express the fate-determining transcription factor, $\mathrm{Nkx} 2.1$ (Xu et al., 2008; Fazzari et al., 2010). Factors that specify chandelier interneuron fate from other Nkx2.1+ lineages, including other PV-expressing interneurons as well as those that express somatostatin, are at this point unknown. During postnatal development, the chandelier axon cartridges begin to be identifiable in mouse cortex at about postnatal day 14, based on analysis of GFP+ cells 
in Nkx2.1Cre MADM mice. In the cat visual cortex, chandelier axon terminals undergo major refinements between the early postnatal period and adulthood, although they appear to target only the AIS (Somogyi et al., 1982).

Interestingly, this postnatal refinement may be quite protracted. In the macaque neocortex, where chandelier cartridges are identifiable by immunohistochemistry for PV (DeFelipe et al., 1989), the detectability of these cartridges is highly dynamic (Anderson et al., 1995). A low density is present by 3 months, but they increase greatly to reach a peak at about 15 months. However, their numbers diminish over the pubertal age range, falling back to the level seen in 3-month-old brains by about 3 years of age. Since neither the density of GABA terminals (Erickson and Lewis, 2002) nor the density of $\mathrm{GABA}_{\mathrm{A}}$ receptors on the AIS (in mice; Katagiri et al., 2007) changes over this period, and since PV expression is known to be dependent on extrinsic factors (Vogt Weisenhorn et al., 1998), the reduction of PV detectability in chandelier axon terminals may reflect alterations of chandelier neuron activity over the pubertal age range. Given the major role of GABA maturation on late-developing aspects of cortical plasticity (Hensch, 2005), evidence that chandelier axon marker expression changes dramatically during postnatal development suggests that it is impor- tant to determine whether the quantity as well as the postsynaptic and network effects of chandelier neuron activity change during the pubertal age range of cortical development.

Clearly, many questions remain regarding the function of ChCs. Even the quite basic question of whether their synapses are depolarizing or hyperpolarizing is still somewhat open. While they may exert different effects in different parts of the brain, this implies a different function for what is otherwise considered a single neuronal subtype, an intriguing possibility. Secondly, if the synapse can be depolarizing in vitro, that does not necessarily hold for conditions in vivo (at least in awake animals), and thus any functions ascribed to ChCs recorded in the slice must be confirmed in the intact brain. Nor has it been established experimentally that the depolarization cortical ChCs can provide is excitatory. The role that ChCs play in controlling or modulating neuronal communication remains an intriguing mystery, hopefully to be solved in the near future.

\section{ACKNOWLEDGMENTS}

We thank laboratory members for comments, Yeonsook Shin and Laura McGarry for help with anatomical reconstructions and histological procedures, and the National Eye Institute and the NIMH for support.

\section{REFERENCES}

Anderson, C. T., Sheets, P. L., Kiritani, T., and Shepherd, G. M. (2010). Sublayerspecific microcircuits of corticospinal and corticostriatal neurons in motor cortex. Nat. Neurosci. 13, 739-744.

Anderson, S. A., Classey, J. D., Conde, F., Lund, J. S., and Lewis, D. A. (1995). Synchronous development of pyramidal neuron dendritic spines and parvalbumin-immunoreactive chandelier neuron axon terminals in layer III of monkey prefrontal cortex. Neuroscience 67, 7-22.

Astman, N., Gutnick,M. J., and Fleidervish, I.A. (2006). Persistent sodium current in layer 5 neocortical neurons is primarily generated in the proximal axon. J. Neurosci. 26, 3465-3473.

Bonifazi, P., Goldin, M., Picardo, M. A., Jorquera, I., Cattani, A., Bianconi, G., Represa, A., Ben-Ari, Y., and Cossart, R. (2009). GABAergic hub neurons orchestrate synchrony in developing hippocampal networks. Science 326, 1419-1424.

Boyden, E. S., Zhang, F., Bamberg, E., Nagel, G., and Deisseroth, K. (2005). Millisecond-timescale, genetically targeted optical control of neural activity. Nat. Neurosci. 8, 1263-1268.
Brecht, M., Schneider, M., Sakmann, B., and Margrie, T. W. (2004). Whisker movements evoked by stimulation of single pyramidal cells in rat motor cortex. Nature 427, 704-710.

Brown, S. P., and Hestrin, S. (2009). Intracortical circuits of pyramidal neurons reflect their long-range axonal targets. Nature 457, 1133-1136.

Cardin, J. A., Carlen, M., Meletis, K., Knoblich, U., Zhang, F., Deisseroth, K., Tsai, L. H., and Moore, C. I. (2009). Driving fast-spiking cells induces gamma rhythm and controls sensory responses. Nature 459, 663-667.

Chattopadhyaya, B., Di Cristo, G., Higashiyama, H., Knott, G. W., Kuhlman, S. J., Welker, E., and Huang, Z. J. (2004). Experience and activitydependent maturation of perisomatic GABAergic innervation in primary visual cortex during a postnatal critical period. J. Neurosci. 24, 9598-9611.

Colbert, C. M., and Pan, E. (2002). Ion channel properties underlying axonal action potential initiation in pyramidal neurons. Nat. Neurosci. 5, 533-538.

De Carlos, J. A., Lopez-Mascaraque, L., and Valverde, F. (1985). Development, morphology and topography of chandelier cells in the auditory cortex of the cat. Brain Res. 354, 293-300.

DeFelipe, J., Hendry, S. H., and Jones, E. G. (1989). Visualization of chandelier cell axons by parvalbumin immunoreactivity in monkey cerebral cortex. Proc. Natl. Acad. Sci. U.S.A. 86 2093-2097.

DeFelipe, J., Hendry, S. H., Jones, E. G. and Schmechel, D. (1985). Variability in the terminations of GABAergic chandelier cell axons on initial segments of pyramidal cell axons in the monkey sensory-motor cortex. J. Comp. Neurol. 231, 364-384.

Destexhe, A., Rudolph, M., and Pare, D. (2003). The high-conductance state of neocortical neurons in vivo. Nat. Rev. Neurosci. 4, 739-751.

Erickson, S. L., and Lewis, D. A. (2002). Postnatal development of parvalbumin- and GABA transporter-immunoreactive axon terminals in monkey prefrontal cortex. J. Comp. Neurol.448, 186-202.

Fairen, A., and Valverde, F. (1980). A specialized type of neuron in the visual cortex of cat: a Golgi and electron microscope study of chandelier cells. J. Comp. Neurol. 194, 761-779.
Farinas, I., and DeFelipe, J. (1991). Patterns of synaptic input on corticocortical and corticothalamic cells in the cat visual cortex. II. The axon initial segment. J. Comp. Neurol. 304, 70-77.

Fazzari, P., Paternain, A. V., Valiente, M., Pla, R., Lujan, R., Lloyd, K., Lerma, J., Marin, O., and Rico, B. (2010). Control of cortical GABA circuitry development by $\mathrm{Nrg} 1$ and ErbB4 signalling. Nature 464, 1376-1380.

Fiumelli, H., Cancedda, L., and Poo, M. M. (2005). Modulation of GABAergic transmission by activity via postsynaptic $\mathrm{Ca} 2+-$ dependent regulation of KCC2 function. Neuron 48, 773-786.

Fleidervish, I. A., Lasser-Ross, N., Gutnick, M. J., and Ross, W. N. (2010). Na+ imaging reveals little difference in action potential-evoked $\mathrm{Na}+$ influx between axon and soma. Nat. Neurosci. 13, 852-860.

Gentet, L. J., Avermann, M., Matyas, F., Staiger, J. F., and Petersen, C. C. (2010). Membrane potential dynamics of GABAergic neurons in the barrel cortex of behaving mice. Neuron 65 , $422-435$. 
Glickfeld, L. L., Roberts, J. D., Somogyi, P., and Scanziani, M. (2009). Interneurons hyperpolarize pyramidal cells along their entire somatodendritic axis. Nat. Neurosci. 12, 21-23.

Gonzalez-Burgos, G., Krimer, L. S., Povysheva, N. V., Barrionuevo, G., and Lewis, D. A. (2005). Functional properties of fast spiking interneurons and their synaptic connections with pyramidal cells in primate dorsolateral prefrontal cortex. J. Neurophysiol. 93, 942-953.

Han, Z. S. (1994). Electrophysiological and morphological differentiation of chandelier and basket cells in the rat hippocampal formation: a study combining intracellular recording and intracellular staining with biocytin. Neurosci. Res. 19, 101-110.

Hasbargen, T., Ahmed, M. M., Miranpuri, G., Li, L., Kahle, K. T., Resnick, D., and Sun, D. (2010). Role of NKCC1 and KCC2 in the development of chronic neuropathic pain following spinal cord injury. Ann. N. Y. Acad. Sci. 1198, 168-172.

Hensch, T. K. (2005). Critical period mechanisms in developing visual cortex. Curr. Top. Dev. Biol. 69, 215-237.

Henze, D. A., and Buzsaki, G. (2001). Action potential threshold of hippocampal pyramidal cells in vivo is increased by recent spiking activity. Neuroscience 105, 121-130.

Houweling, A. R., and Brecht, M. (2008). Behavioural report of single neuron stimulation in somatosensory cortex. Nature 451, 65-68.

Hu, W., Tian, C., Li, T., Yang, M., Hou, H., and Shu, Y. (2009). Distinct contributions of $\mathrm{Na}(\mathrm{v}) 1.6$ and $\mathrm{Na}(\mathrm{v}) 1.2$ in action potential initiation and backpropagation. Nat. Neurosci. 12, 996-1002.

Inda, M. C., Defelipe, J., and Munoz, A. (2007). The distribution of chandelier cell axon terminals that express the GABA plasma membrane transporter GAT-1 in the human neocortex. Cereb. Cortex 17, 2060-2071.

Jones, E. G. (1975). Varieties and distribution of non-pyramidal cells in the somatic sensory cortex of the squirrel monkey. J. Comp. Neurol. 160, 205-267.

Kantrowitz, J. T., Francis, N. N., Salah, A., and Perkins, K. L. (2005). Synaptic depolarizing GABA Response in adults is excitatory and proconvulsive when GABAB receptors are blocked. J. Neurophysiol. 93, 2656-2667.

Katagiri, H., Fagiolini, M., and Hensch, T. K. (2007). Optimization of somatic inhibition at critical period onset in mouse visual cortex. Neuron 53 805-812.

Kawaguchi, Y. (1995). Physiological subgroups of nonpyramidal cells with specific morphological characteristics in layer II/III of rat frontal cortex. J. Neurosci. 15, 2638-2655.

Khirug, S., Yamada, J., Afzalov, R., Voipio, J., Khiroug, L., and Kaila, K. (2008) GABAergic depolarization of the axon initial segment in cortical principal neurons is caused by the $\mathrm{Na}-\mathrm{K}-2 \mathrm{Cl}$ cotransporter NKCC1. J. Neurosci. 28, 4635-4639.

Klausberger, T., Magill, P. J., Marton, L. F., Roberts, J. D., Cobden, P. M., Buzsaki, G., and Somogyi, P. (2003). Brainstate- and cell-type-specific firing of hippocampal interneurons in vivo. Nature 421, 844-848.

Kyrozis, A., and Reichling, D. B. (1995). Perforated-patch recording with gramicidin avoids artifactual changes in intracellular chloride concentration. J. Neurosci. Methods 57, 27-35.

Lewis, D. A., Foote, S. L., and Cha, C. I. (1989). Corticotropin-releasing factor immunoreactivity in monkey neocortex: an immunohistochemical analysis. J. Comp. Neurol. 290, 599-613.

Lorente de Nó, R. (1922). La corteza cerebral del ratón. Trab. Lab. Invest. Bio. (Madrid) 20, 41-78.

Markram, H., Toledo-Rodriguez, M., Wang, Y., Gupta, A., Silberberg, G., and $\mathrm{Wu}, \mathrm{C}$. (2004). Interneurons of the neocortical inhibitory system. Nat. Rev. Neurosci. 5, 793-807.

Marr, D. (1970). A theory for cerebral neocortex. Proc. R. Soc. Lond., B, Biol. Sci. 176, 161-234.

Marshel, J. H., Mori, T., Nielsen, K. J., and Callaway, E. M. (2010). Targeting single neuronal networks for gene expression and cell labeling in vivo. Neuron 67, 562-574.

McDonald, A. J. (1982). Neurons of the lateral and basolateral amygdaloid nuclei: a Golgi study in the rat. J. Comp. Neurol. 212, 293-312.

Meyer, A. H., Katona, I., Blatow, M., Rozov, A., and Monyer, H. (2002). In vivo labeling of parvalbumin-positive interneurons and analysis of electrical coupling in identified neurons. $J$. Neurosci. 22, 7055-7064.

Miles, R., Toth, K., Gulyas, A. I., Hajos, N. and Freund, T. F. (1996). Differences between somatic and dendritic inhibition in the hippocampus. Neuron 16, 815-823.

Molnar, G., Olah, S., Komlosi, G., Fule, M., Szabadics, J., Varga, C., Barzo, P., and Tamas, G. (2008). Complex events initiated by individual spikes in the human cerebral cortex. PLoS
Biol. 6, e222. doi: 10.1371/journal. pbio.0060222.

Murayama, M., Perez-Garci, E., Nevian, T., Bock, T., Senn, W., and Larkum, M. E. (2009). Dendritic encoding of sensory stimuli controlled by deep cortical interneurons. Nature 457, 1137-1141.

Nikolenko, V., Poskanzer, K. E., and Yuste, R. (2007). Two-photon photostimulation and imaging of neural circuits. Nat. Methods 4 943-950.

Perkins, K. L. (2006). Cell-attached voltage-clamp and current-clamp recording and stimulation techniques in brain slices. J. Neurosci. Methods 154, 1-18.

Poulet, J. F., and Petersen, C. C. (2008) Internal brain state regulates membrane potential synchrony in barrel cortex of behaving mice. Nature 454, 881-885.

Ramón y Cajal, S. (1899). La Textura del Sistema Nerviosa del Hombre y los Vertebrados. Madrid: Moya (Primera Edicion).

Rheims, S., Minlebaev, M., Ivanov, A., Represa, A., Khazipov, R., Holmes, G. L., Ben-Ari, Y., and Zilberter, Y. (2008) Excitatory GABA in rodent developing neocortex in vitro. J. Neurophysiol. 100, 609-619.

Sik, A., Tamamaki, N., and Freund, T. F. (1993). Complete axon arborization of a single CA3 pyramidal cell in the rat hippocampus, and its relationship with postsynaptic parvalbumin-containing interneurons. Eur. J. Neurosci. 5, 1719-1728.

Smetters, D., Majewska, A., and Yuste, R. (1999). Detecting action potentials in neuronal populations with calcium imaging. Methods 18, 215-221.

Sohal, V. S., Zhang, F., Yizhar, O., and Deisseroth, K. (2009). Parvalbumin neurons and gamma rhythms enhance cortical circuit performance. Nature 459, 698-702.

Somogyi,P.(1977).A specific"axo-axonal" interneuron in the visual cortex of the rat. Brain Res. 136, 345-350.

Somogyi, P., Freund, T. F., and Cowey, A. (1982). The axo-axonic interneuron in the cerebral cortex of the rat, cat and monkey. Neuroscience 7 2577-2607.

Somogyi, P., Freund, T. F., Hodgson, A. J., Somogyi, J., Beroukas, D., and Chubb, I.W. (1985). Identified axo-axonic cells are immunoreactive for GABA in the hippocampus and visual cortex of the cat. Brain Res. 332, 143-149.

Somogyi, P., Nunzi, M. G., Gorio, A., and Smith, A. D. (1983). A new type of specific interneuron in the monkey hippocampus forming synapses exclusively with the axon initial seg- ments of pyramidal cells. Brain Res. 259, 137-142.

Soriano, E., and Frotscher, M. (1989). A GABAergic axo-axonic cell in the fascia dentata controls the main excitatory hippocampal pathway. Brain Res. 503, 170-174

Szabadics, J., Varga, C., Molnar, G., Olah, S., Barzo, P., and Tamas, G. (2006). Excitatory effect of GABAergic axoaxonic cells in cortical microcircuits. Science 311, 233-235.

Szentagothai,J. (1975). The "module-concept" in cerebral cortex architecture. Brain Res. 95, 475-496.

Tukker, J. J., Fuentealba, P., Hartwich, K., Somogyi, P., and Klausberger, T. (2007). Cell type-specific tuning of hippocampal interneuron firing during gamma oscillations in vivo. J. Neurosci. 27, 8184-8189.

Valeeva, G., Abdullin, A., Tyzio, R., Skorinkin, A., Nikolski, E., Ben-Ari, Y., and Khazipov, R. (2010). Temporal coding at the immature depolarizing GABAergic synapse. Front. Cell. Neurosci. 4:12. doi: 10.3389/ fncel.2010.00017.

Varga, C., Lee, S. Y., and Soltesz, I. (2010). Target-selective GABAergic control of entorhinal cortex output. Nat. Neurosci. 13, 822-824.

Vida, I., Bartos, M., and Jonas, P. (2006). Shunting inhibition improves robustness of gamma oscillations in hippocampal interneuron networks by homogenizing firing rates. Neuron 49, 107-117.

Vogt Weisenhorn, D. M., Celio, M. R., and Rickmann, M. (1998). The onset of parvalbumin-expression in interneurons of the rat parietal cortex depends upon extrinsic factor(s). Eur. J. Neurosci. 10, 1027-1036.

Wickersham, I. R., Lyon, D. C., Barnard, R. J., Mori, T., Finke, S., Conzelmann, K. K., Young, J. A., and Callaway, E. M. (2007). Monosynaptic restriction of transsynaptic tracing from single, genetically targeted neurons. Neuron 53, 639-647.

Woodin, M. A., Ganguly, K., and Poo, M. M. (2003). Coincident pre- and postsynaptic activity modifies GABAergic synapses by postsynaptic changes in Cl- transporter activity. Neuron 39, 807-820.

Woodruff,A., Xu, Q., Anderson, S.A., and Yuste, R. (2009). Depolarizing effect of neocortical chandelier neurons. Front. Neural Circuits 3:15. doi: 10.3389/ neuro.04.015.2009.

Woodruff, A. R., Monyer, H., and Sah, P. (2006). GABAergic excitation in the basolateral amygdala. J. Neurosci. 26, 11881-11887.

Xu, Q., Tam, M., and Anderson, S. A. (2008). Fate mapping Nkx2.1-lineage 
cells in the mouse telencephalon. $J$. Comp. Neurol. 506, 16-29.

Xu, X., and Callaway, E. M. (2009). Laminar specificity of functional input to distinct types of inhibitory cortical neurons. J. Neurosci. 29, 70-85.

Yoshimura, Y., Dantzker, J. L., and Callaway, E. M. (2005). Excitatory cortical neurons form fine-scale functional networks. Nature 433, 868-873.
Yuste, R., and Katz, L. C. (1991). Control of postsynaptic Ca2+ influx in developing neocortex by excitatory and inhibitory neurotransmitters. Neuron 6, 333-344.

Zhu, Y., Stornetta, R. L., and Zhu, J. J. (2004). Chandelier cells control excessive cortical excitation: characteristics of whisker-evoked synaptic responses of layer $2 / 3$ nonpyramidal and pyramidal neurons. J. Neurosci. 24, 5101-5108.
Conflict of Interest Statement: The authors declare that the research was conducted in the absence of any commercial or financial relationships that could be construed as a potential conflict of interest.

Received:20August2010; accepted: 19November 2010; published online: 08 December 2010. Citation: Woodruff AR, Anderson SA and Yuste R (2010) The enigmatic function of chandelier cells. Front. Neurosci. 4:201. doi 10.3389/fnins.2010.00201

Copyright $\odot 2010$ Woodruff, Anderson and Yuste. This is an open-access article subject to an exclusive license agreement between the authors and the Frontiers Research Foundation, which permits unrestricted use, distribution, and reproduction in any medium, provided the original authors and source are credited. 